\title{
COMPUTACIONAL STUDY OF THE ELECTRONIC EFFECTS IN ROTATIONAL BARRIER OF THE N-ARILCARBAMATOS N-CO BOND
}

\author{
GRASEL, Fábio dos Santos ${ }^{1 *} ;$ FONTOURA, Luiz Antonio Mazzini² \\ ${ }^{1}$ Pontifícia Universidade Católica do Rio Grande do Sul, Programa de Pós-graduação em Engenharia e \\ Tecnologia de Materiais, Departamento de Química, Av Ipiranga, 6681, cep 90619-900, Porto Alegre - RS, \\ Brasil \\ (fone: +55 513632 4055; fax: +55 513632 2863) \\ ${ }^{2}$ Universidade Luterana do Brasil, Curso de Química, Rua Farroupilha, 8001, 92425-900 Canoas - RS, Brasil \\ (fone: +55 513477 9163; fax: +55 513477 1313) \\ ${ }^{*}$ Autor correspondente \\ e-mail: fsgrasel@gmail.com
}

\section{RESUMO}

Received 21 June 2015; received in revised form 03 December 2015; accepted 04 December 2015

Amidas são alvos de estudo particularmente importantes uma vez que suas propriedades interessam a áreas que vão da bioquímica à ciência de materiais, basta observar que a ligação entre o nitrogênio e a carbonila que está presente em polipeptídios naturais como as proteínas, e sintéticos, como as poliamidas. Amidas e derivados apresentam, em geral, duas geometrias de equilíbrio, resultado da barreira energética envolvida no processo rotacional da ligação entre o nitrogênio e a carbonila, sendo este influenciado por fatores eletrônicos e estéreos. Neste aspecto, amidas e carbamatos derivados da anilina são substratos particularmente interessantes, uma vez que efeitos eletrônicos e estéreos podem ser avaliados através de substituintes apropriados no anel ou no nitrogênio. Neste trabalho, o mecanismo rotacional da ligação entre o nitrogênio e da carbonila de $\mathrm{N}$-arilcarbamatos foi estudado por modelagem molecular através do semi-empírico AM1 e do ab initio HF/6-31G*. A análise conformacional forneceu duas geometrias de equilíbrio (E e Z) e duas conformações de máxima energia, tomadas como aproximações dos estados de transição $E T_{\text {syn }}$ e $E T_{\text {anti. }} \mathrm{O}$ cálculo ab initio estima as barreiras na faixa entre 13 a $17 \mathrm{kcal}^{\mathrm{mol}}{ }^{-1}$. Resultados semi-empíricos demonstraram uma relação de aproximadamente $40 \%$ do valor da barreira rotacional em relação aos ab initio.

Palavras-chave: Carbamato, Ligação amídica, Equilíbrio conformacional.

\section{ABSTRACT}

Amides are the subject of many particularly important studies, as their properties are of interest to different areas, spanning from biochemistry to material sciences because this functional group is present, for instance, in both natural and artificial polymers such as proteins and polyamides. Amides and their derivatives show, frequently, two different equilibrium geometries, which result from an energetic barrier involved on the $\mathrm{N}$ $\mathrm{CO}$ bond rotation. The rotational mechanism is influenced by electronic and steric effects. In this sense, amides and carbamates from aniline are particularly interesting substrates, since those effects can be evaluated through aryl or nitrogen substituents. In this work, the $\mathrm{N}$-arylcarbamates $\mathrm{N}-\mathrm{CO}$ rotational mechanisms were studied by AM1 semi-empiric and HF/6-31G* ab initio calculation. The conformational analysis showed, as expected, two equilibrium geometries and two maximum energies rotamers (transition states $\mathrm{TS}_{\text {syn }}$ and $\mathrm{TS}_{\text {anti }}$ ). The rotational barriers were calculated by the energies differences between transition states and equilibrium geometries. Barriers were estimated at $13-17 \mathrm{kcal}^{\mathrm{mol}}{ }^{-1}$ by the $\mathrm{HF} / 6-31 \mathrm{G}^{*}$ calculation. AM1 results, by the other side, are about $40 \%$ from the ab initio ones.

Keywords: Carbamate, Amidic bond, Conformational equilibrium.

PERIÓDICO TCHÊ QUÍMICA • www.periodico.tchequimica.com • Vol. 13 N. 25.

- ISSN 1806-0374 (impresso) • ISSN 1806-9827 (CD-ROM) • ISSN 2179-0302 (meio eletrônico)

(C) 2015. Porto Alegre, RS. Brasil 


\section{INTRODUÇÃO}

Amidas são alvos de estudo particularmente importantes uma vez que suas propriedades interessam a áreas que vão da bioquímica à ciência de materiais (Liang et al. 2016; Moreno et al. 2016; Sun et al. 2016). Basta observar que a ligação entre o nitrogênio e a carbonila está presente em polipeptídios naturais como as proteínas, e sintéticos, como as poliamidas (Avalos et al., 2001; Montazer and Nia, 2015; 2. Smith et al., 2004). Mizushima e colaboradores (1950) mostraram experimentalmente a existência de uma barreira rotacional entre a ligação nitrogênio e a carbonila da $\mathrm{N}$-metilacetamida e a predominância da forma trans. Quando as amidas assumem suas geometrias de mais baixa energia, o nitrogênio e seus grupos são coplanares. A ligação entre o nitrogênio e a carbonila é mais curta do que nas aminas e sua rotação é lenta, o que tem sido atribuído ao caráter parcial de dupla ligação imposta pela ressonância do par de elétrons isolados do nitrogênio e a carbonila (Avalos et al., 2001; Fischer, 2000; Fontoura et al., 2002; Gálvez et al., 2015; Lauvergnat e Hiberty, 1997) conforme apresentado no esquema 1.

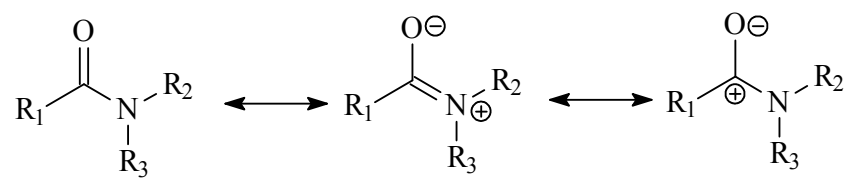

a b

c

\section{Esquema 1. Estruturas de ressonância de amidas}

O caráter parcial de dupla ligação faz surgirem duas geometrias de equilíbrio. Durante um processo de rotação da ligação entre o nitrogênio e a carbonila, um giro de aproximadamente $90^{\circ}$ leva a conformações de máxima energia (Fischer, 2000; Fontoura et al., 2002; Wiberg e Breneman, 1992), que correspondem aos estados de transição no processo de interconversão de uma geometria de equilíbrio na outra. Essas conformações de

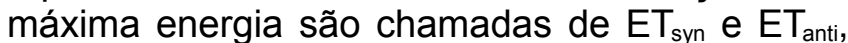
nas quais o par de elétrons isolado do nitrogênio assume, respectivamente, orientação syn e antiperiplanar ao oxigênio da carbonila (Fontoura et al., 2002).
Qualquer que seja o sentido da rotação observa-se a diminuição da ressonância, com passagem do nitrogênio trigonal-planar (hibridização $s p^{2}$ ) das geometrias de equilíbrio para piramidal (hibridização $s p^{3}$ ) nos estados de transição, além de um aumento do comprimento da ligação entre o nitrogênio e a carbonila, resultado de uma menor participação de sua forma canônica b (Fontoura et al., 2002), (esquema 1).

Muitos experimentos e estudos teóricos da estereoquímica cis e trans da ligação amídica têm sido feitos (Gálvez et al., 2015; Saito et al., 1995). A estereoquímica desses compostos é muitas vezes crítica para a determinação de suas atividades biológicas, o que é de grande interesse na química medicinal (Saito et al., 1995).

Recente desenvolvimento em pesquisa química destaca um crescimento no uso dos métodos computacionais no estudo de estruturas moleculares e reações químicas. O crescimento contínuo na exatidão dos resultados computacionais oferece novas oportunidades para calcular o papel de vários fatores moleculares na reatividade e estabilidade conformacional de grupos funcionais (llieva et al. 2002; Latelli et al. 2014; Ortega et al. 2015).

O equilíbrio conformacional da $\mathrm{N}$ metilformamida e da $\mathrm{N}$-metilacetamida encontrase deslocado no sentido do confôrmero $Z$, o que pode ser explicado por fatores estéreos e pela interação de carga entre a carbonila e a metila ligada ao nitrogênio (Eliel e Wilen, 1994).

Wiberg e Breneman (1992) calcularam a barreira rotacional da formamida pelo método computacional MP2/6-31G* $\left(16,7 \mathrm{kcal}^{\mathrm{mol}}{ }^{-1}\right)$. Em trabalho posterior realizado por Gálvez et al. (2015) calcularam a barreira rotacional da formamida pelos métodos computacionais B3LYP/6-311++G(2d,2p), MP2/6-311++G(2d,2p) and $\operatorname{CCSD}(\mathrm{T}) / 6-311++\mathrm{G}(2 \mathrm{~d}, 2 \mathrm{p}) / / \mathrm{B} 3 \mathrm{LYP} / 6-311+$ $+\mathrm{G}(2 \mathrm{~d}, 2 \mathrm{p})$, obtendo resultados de 18,20 a 18,69 $\left.\mathrm{kcal} . \mathrm{mol}^{-1}\right)$. Estes resultados apresentaram boa concordância com os resultados experimentais (16,6 a $19 \mathrm{kcal}^{\mathrm{mol}}{ }^{-1}$ ) (Taha et al., 1998; Wiberg e Breneman, 1992).

Segundo Fisher (2000) substituintes ligados à carbonila ou ao nitrogênio de uma amida influenciam na energia da barreira rotacional. Um substituinte produz efeitos 
diferentes conforme esteja ligado ao nitrogênio ou a carbonila. Fatores estéreos são mais significativos na contribuição da barreira rotacional, conforme o volume dos substituintes que estão ligados ao nitrogênio ou a carbonila.

Substituintes eletrodoadores ligados à carbonila baixam 0 valor da barreira, pois estabilizam parcialmente este grupo que é eletronegativo, diminuindo assim a afinidade pelo par de elétrons isolados do nitrogênio. $O$ efeito contrário pode ser notado quando um grupo eletrodoador está ligado ao nitrogênio, pois este estabiliza a carga parcial positiva, deixando o par de elétrons isolados deste grupo ainda mais disponível para a ressonância.

Saito e colaboradores (1995) estudaram por cálculos de orbital molecular ab initio (Hartree-Fock) a estabilidade de acetanilidas e $\mathrm{N}$-metilacetanilidas, constatando que, no caso das primeiras, a forma trans é mais estável que a forma cis por 2,15 kcal.mol-1, enquanto que, nas últimas, a forma cis é mais estável que a trans por $3,50 \mathrm{kcal} . \mathrm{mol}^{-1}$ no conjunto de bases 6$31 \mathrm{G}^{* *} / / 4-31 \mathrm{G}$. O autor utiliza a denominação cis e trans com respeito às posições relativas dos grupos alquila e arila. No caso da acetanilida os dois grupos mais volumosos (metil e fenil), assumem posição trans, tornando menor o impedimento espacial. Assim o anel mantém a planaridade com 0 nitrogênio, dando maior estabilidade por ressonância. Já no caso da $N$ metilacetanilida, os dois grupos metilas assumem posição trans e o anel aromático forma um ângulo de aproximadamente $90^{\circ} \mathrm{com}$ a ligação do nitrogênio com a carbonila, diminuindo dessa forma o impedimento espacial.

Em outro trabalho, llieva e colaboradores (2002) afirmam baseados em resultados experimentais que a forma trans de amidas e uréias é normalmente a termodinamicamente mais estável. Resultados teóricos obtidos por TFD (teoria do funcional de densidade) em uma série de acetanilidas secundárias estudadas indicam uma diferença de 2,62 a 4,15 kcal. mol-1 entre a forma cis e a forma trans, com energia mais baixa para os confôrmeros trans em todos os casos.

Carbamatos, apesar da variada atividade biológica, têm sido relativamente menos estudados do que as amidas. Como os carbamatos são compostos de função mista, amida e éster é interessante incluir este último na discussão da análise conformacional (Fontoura et al., 2002).

Assim como as amidas, os ésteres também possuem duas geometrias de equilíbrio E e Z, conforme apresentado no esquema 2.<smiles>[R]OC([R])=O</smiles>
Z

E

Esquema 2. Equilíbrio conformacional de derivados de ácidos carboxílicos.

Souza e colaboradores (1996) em seus trabalhos de pesquisa constataram uma diferença de energia muito grande entre os confôrmeros $E$ e $Z$, com deslocamento do equilíbrio no sentido deste último. Estes fatores podem ser explicados em termos de interações estéreas do grupo R com R', pois esta repulsão desestabiliza a conformação $E$, principalmente quando o grupo R' é um grupo volumoso (Pawar eta I., 1998). Outro fator considerável é a interação eletrostática entre o oxigênio da carbonila, rico em elétrons e o grupo $R^{\prime}$ que tem baixa densidade eletrônica (Fontoura et al., 2002). Tornado assim a conformação $E$ praticamente negligenciável a temperatura ambiente (Eliel e Wilen, 1994).

Os carbamatos, assim como as amidas e os ésteres, apresentam a estrutura nitrogênio, carbonila e oxigênio planar ou aproximadamente planar, com o nitrogênio $s p^{2}$.

Souza et al. (1996) realizaram cálculos ab initio $\left(\mathrm{HF} / 3-21 \mathrm{G}\left(^{*}\right)\right)$ para o $\mathrm{N}, \mathrm{N}$-dimetilcarbamato de metila evidenciando a estabilidade do confôrmero $Z$ e sua completa planaridade, com geometria similar à determinada por Raio-X. $\mathrm{O}$ rotâmero $\mathrm{E}$ é desfavorecido devido à repulsão estérica entre os grupos metilas ligados ao nitrogênio e ao oxigênio. Observa-se que no caso do carbamato estudado por Souza et al. (1996) uma rotação de $180^{\circ}$ da ligação entre o nitrogênio e a carbonila não altera a estrutura do composto. A rotação da ligação entre o oxigênio e a carbonila, por sua vez, faz surgirem os dois rotâmeros. Da mesma forma como ocorre nos ésteres, o equilíbrio encontra-se fortemente deslocado no sentido a geometria Z. Assim como nas amidas, os carbamatos, durante a rotação da 
ligação entre o nitrogênio e a carbonila, também

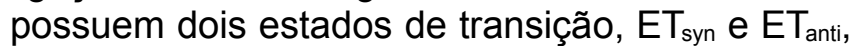
sendo que neste caso a energia do primeiro é 1,6 kcal.mol-1 menor, de acordo com os cálculos realizados (MP2/3-21G $\left.{ }^{*}\right) / / \mathrm{HF} / 6-31 \mathrm{G}^{*}$ ) (Souza et al., 1996). Segundo o autor, este último possui maior repulsão dos pares isolados do nitrogênio e do oxigênio $\alpha$-carbonílico, tornando-o, desta forma, mais instável que o primeiro. A barreira rotacional, para a rotação da ligação entre o nitrogênio e a carbonila foi estimada em 16,1 kcal. $\mathrm{mol}^{-1}$.

Deetz et al., (2002) estudaram por método experimental a diferença da barreira rotacional de amidas e carbamatos constatando que a barreira rotacional de $\mathrm{N}$-alquilcarbamatos era normalmente em torno de $16 \mathrm{kcal}_{\mathrm{mol}}{ }^{-1}$, sendo cerca de 1 a $4 \mathrm{kcal}^{\mathrm{mol}}{ }^{-1}$ mais baixo que as amidas estruturalmente relacionadas. No caso dos $\mathrm{N}$-fenilcarbamatos a barreira rotacional é mais baixa, em torno de $12,5 \mathrm{kcal}^{\mathrm{mol}}{ }^{-1}$. Este efeito é atribuído à competição do anel com a carbonila pelo par de elétrons isolados do nitrogênio.

Neste trabalho, foram determinados de modo teórico, os valores das barreiras rotacionais de $\mathrm{N}$-arilcarbamatos parasubstituídos, bem como o efeito eletrônico dos grupos no anel aromático sobre o valor da barreira.

\section{METODOLOGIA}

Realizaram-se otimizações de geometria para as conformações de diversos $N$ arilcarbamatos para-substituídos, conforme o Esquema 3, através de cálculo semi-empírico AM1 (Dewar et al., 1985), utilizando o programa Pc Spartan Pro 1.0.5 (Wavefunction, 2000). Esses compostos foram otimizados para ângulos de diedro $\mathrm{C}_{4}-\mathrm{N}_{3}-\mathrm{C}_{2}-\mathrm{O}_{1}$, restritos de 0 a $360^{\circ}$ com incrementos de $10^{\circ}$. Os dois confôrmeros mais estáveis foram obtidos através da otimização de geometria dos rotâmeros de menor energia, liberados de qualquer restrição.

Destes cálculos obtiveram-se os seguintes parâmetros de geometria: ângulo das ligações $\mathrm{C}_{4}-\mathrm{N}_{3}-\mathrm{C}_{2}$ (ângulo $\alpha$ ), $\mathrm{C}_{2}-\mathrm{N}_{3}-\mathrm{R}_{1}$ (ângulo $\beta$ ) e $\mathrm{C}_{4}-\mathrm{N}_{3}-\mathrm{R}_{1}$ (ângulo $\mathrm{Y}$ ); comprimentos das ligações $\mathrm{O}_{1}-\mathrm{C}_{2}, \mathrm{C}_{2}-\mathrm{N}_{3}$ e $\mathrm{N}_{3}-\mathrm{C}_{4}$ e diedros $\mathrm{O}_{1}-\mathrm{C}_{2}-\mathrm{N}_{3}-\mathrm{C}_{4}$ e
$\mathrm{C}_{5}-\mathrm{C}_{4}-\mathrm{N}_{3}-\mathrm{C}_{2}$. Além dos parâmetros geométricos, buscou-se o calor de formação $\left(\Delta \mathrm{H}_{\mathrm{f}}\right)$.

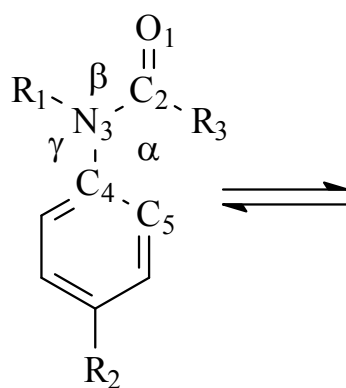

$Z$

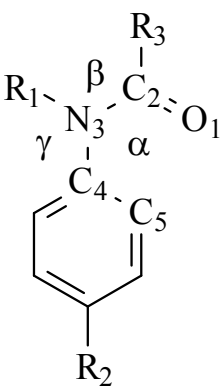

E
$1 \mathrm{R}_{1}=\mathrm{H}, \mathrm{R}_{2}=\mathrm{CH}_{3} \mathrm{O}, \mathrm{R}_{3}=t-\mathrm{BuO}$

$2 \mathrm{R}_{1}=\mathrm{H}, \mathrm{R}_{2}=\mathrm{CH}_{3}, \mathrm{R}_{3}=t-\mathrm{BuO}$

$3 \mathrm{R}_{1}=\mathrm{H}, \mathrm{R}_{2}=\mathrm{H}, \mathrm{R}_{3}=t-\mathrm{BuO}$

$4 \mathrm{R}_{1}=\mathrm{H}, \mathrm{R}_{2}=\mathrm{Cl}, \mathrm{R}_{3}=t-\mathrm{BuO}$

$5 \mathrm{R}_{1}=\mathrm{H}, \mathrm{R}_{2}=\mathrm{NO}_{2}, \mathrm{R}_{3}=t-\mathrm{BuO}$

$6 \mathrm{R}_{1}=\mathrm{CH}_{3}, \mathrm{R}_{2}=\mathrm{CH}_{3} \mathrm{O}, \mathrm{R}_{3}=t-\mathrm{BuO}$

$7 \mathrm{R}_{1}=\mathrm{CH}_{3}, \mathrm{R}_{2}=\mathrm{CH}_{3}, \mathrm{R}_{3}=t-\mathrm{BuO}$

$8 \mathrm{R}_{1}=\mathrm{CH}_{3}, \mathrm{R}_{2}=\mathrm{H}, \mathrm{R}_{3}=t-\mathrm{BuO}$

$9 \mathrm{R}_{1}=\mathrm{CH}_{3}, \mathrm{R}_{2}=\mathrm{Cl}, \mathrm{R}_{3}=t-\mathrm{BuO}$

$10 \mathrm{R}_{1}=\mathrm{CH}_{3}, \mathrm{R}_{2}=\mathrm{NO}_{2}, \mathrm{R}_{3}=t-\mathrm{BuO}$

$11 \mathrm{R}_{1}=\mathrm{H}, \mathrm{R}_{2}=\mathrm{CH}_{3} \mathrm{O}, \mathrm{R}_{3}=\mathrm{CH}_{3} \mathrm{O}$

$12 \mathrm{R}_{1}=\mathrm{H}, \mathrm{R}_{2}=\mathrm{CH}_{3}, \mathrm{R}_{3}=\mathrm{CH}_{3} \mathrm{O}$

$13 \mathrm{R}_{1}=\mathrm{H}, \mathrm{R}_{2}=\mathrm{H}, \mathrm{R}_{3}=\mathrm{CH}_{3} \mathrm{O}$

$14 \mathrm{R}_{1}=\mathrm{H}, \mathrm{R}_{2}=\mathrm{Cl}, \mathrm{R}_{3}=\mathrm{CH}_{3} \mathrm{O}$

$15 \mathrm{R}_{1}=\mathrm{H}, \mathrm{R}_{2}=\mathrm{NO}_{2}, \mathrm{R}_{3}=\mathrm{CH}_{3} \mathrm{O}$

$16 \mathrm{R}_{1}=\mathrm{CH}_{3}, \mathrm{R}_{2}=\mathrm{CH}_{3} \mathrm{O}, \mathrm{R}_{3}=\mathrm{CH}_{3} \mathrm{O}$

$17 \mathrm{R}_{1}=\mathrm{CH}_{3}, \mathrm{R}_{2}=\mathrm{CH}_{3}, \mathrm{R}_{3}=\mathrm{CH}_{3} \mathrm{O}$

$18 \mathrm{R}_{1}=\mathrm{CH}_{3}, \mathrm{R}_{2}=\mathrm{H}, \mathrm{R}_{3}=\mathrm{CH}_{3} \mathrm{O}$

$19 \mathrm{R}_{1}=\mathrm{CH}_{3}, \mathrm{R}_{2}=\mathrm{Cl}, \mathrm{R}_{3}=\mathrm{CH}_{3} \mathrm{O}$

$20 \mathrm{R}_{1}=\mathrm{CH}_{3}, \mathrm{R}_{2}=\mathrm{NO}_{2}, \mathrm{R}_{3}=\mathrm{CH}_{3} \mathrm{O}$

Esquema 3. Equilíbrio conformacional de derivados de ácidos carboxílicos. Compostos estudados e seus ângulos de ligações $\alpha, \beta$ e $\gamma$.

Após, os dados das estruturas mais estáveis (conformações de equilíbrio) e das mais instáveis (estados de transição) foram utilizados como dados de entrada, para cálculos ab initio Hartree-Fock no conjunto de base 6-31G*. Em alguns casos foi observada a inversão de configuração do nitrogênio no estado de transição, nestas situações o diedro imediatamente anterior foi tomado como novo estado de transição.

As barreiras rotacionais foram estimadas através das diferenças de calores de formação (no método AM1) ou energia total da molécula $\left(\mathrm{HF} / 6-31 \mathrm{G}^{*}\right)$ entre os estados de transição (considerados como as conformações de máxima energia encontradas durante a análise 
conformacional) e as conformações de equilíbrio (rotâmeros de menor energia).

\section{RESULTADOS E DICUSSÃO}

A Figura 1 apresenta a variação de energia do $N$-metil- $N$-( $p$-tolil)carbamato de tercbutila (8) à medida que ocorre o giro da ligação entre o nitrogênio e a carbonila. Conforme o esperado, a análise conformacional mostrou duas geometrias de máxima e duas de mínima energia. Este comportamento se reproduz para todos os demais compostos estudados.

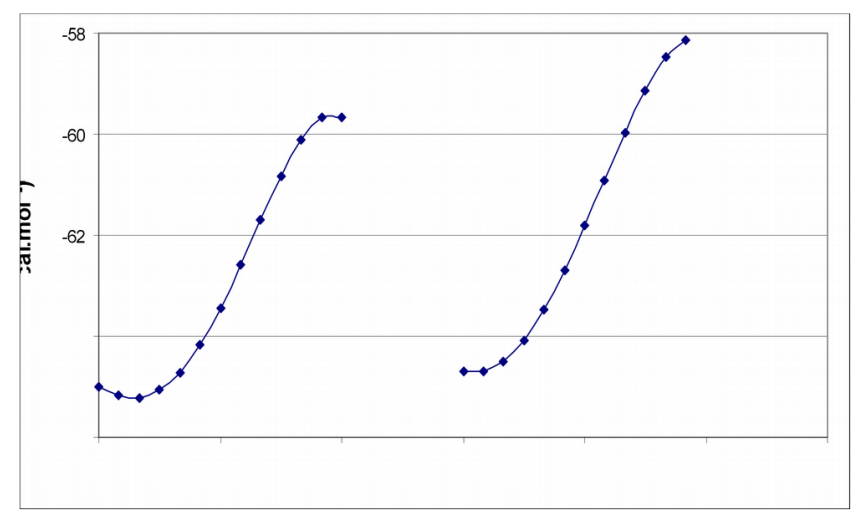

Figura 1. Calor de formação $\left(\mathrm{kcal}^{\mathrm{mol}}{ }^{-1}\right) \mathrm{x}$ diedro (graus): valores obtidos para o $N$-metil- $N-(p-$ tolil)carbamato de terc-butila (8), através de cálculo semi-empírico AM1 (PcSpartan Pro, versão 1.0.5).

A curva apresenta descontinuidade devido à inversão do nitrogênio nas proximidades dos estados de transição. A inversão do nitrogênio modifica a orientação do seu par isolado com relação à carbonila de syn para antiperiplanar em $\mathrm{ET}_{\text {syn }}$, conforme Esquema 4, e o contrário em $\mathrm{ET}_{\text {anti. }}$ Por esta razão, estes pontos foram desprezados.<smiles>[R]C1N2CCC3(c4ccccc43)C1([R])CCC2=O</smiles><smiles>[R3]C1CCC2([R])CC1CN(Cc1ccccc1)C2=O</smiles>

Esquema 4. Inversão de configuração do nitrogênio de syn-periplanar para anti-periplanar.

À esquerda da Fgura 2, é apresentada a conformação com diedro de $120^{\circ}$ para o $\mathrm{N}$-metil$N$-(p-tolil)carbamato de terc-butila (8).

Um incremento de $10^{\circ}$, ao invés de levar ao diedro de $130^{\circ}$, forneceu a conformação mostrada na direita, na qual se observa a pirâmide do nitrogênio invertida. Esta conformação nada mais é, que a imagem especular do diedro de $230^{\circ}$, apresentando desta forma, as mesmas propriedades do que essa conformação e não os valores reais para o diedro de $130^{\circ}$ (Fontoura et al., 2002).

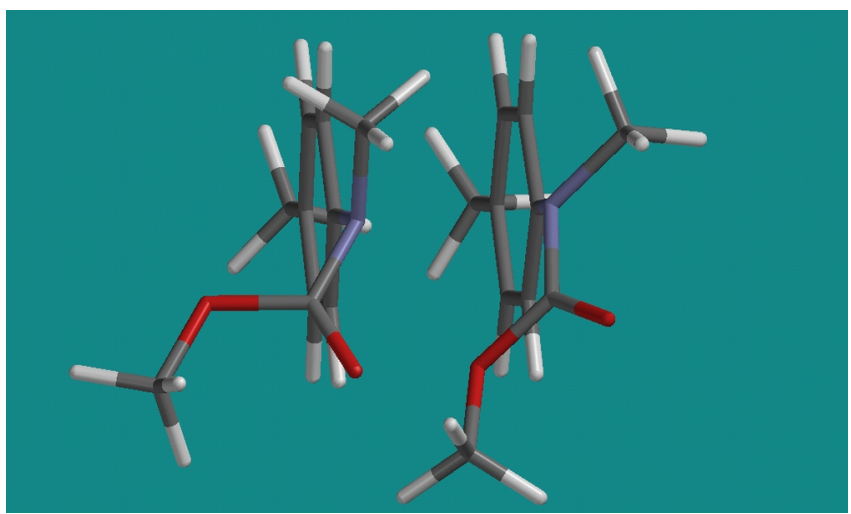

Figura 2. Inversão da pirâmide do nitrogênio em $N$-metil-N-(p-tolil)carbamato de terc-butila (8), realizado por cálculo semi-empírico AM1.

A geometria do nitrogênio nas conformações de equilíbrio e nos estados de transição pode ser avaliada através do somatório dos seus ângulos de ligação (ângulos $\alpha, \beta$ e $\gamma$ ). A Figura 3 apresenta os somatórios em função do diedro obtidos para o $N$-metil- $N$ - $(p$-tolil)carbamato de terc-butila (8).

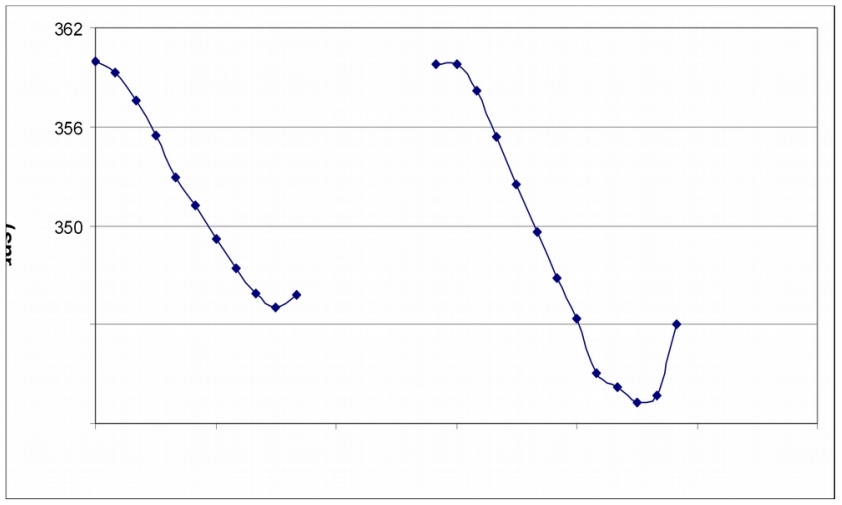

Figura 3. Somatório dos ângulos de ligação em torno do nitrogênio (graus) x diedro (graus): valores obtidos para o $N$-metil- $N$ - $(p$ tolil)carbamato de terc-butila (8), através de cálculo semi-empírico AM1 (PcSpartan Pro, versão 1.0.5).

Nas geometrias de equilíbrio, o somatório

PERIÓDICO TCHÊ QUÍMICA • www.periodico.tchequimica.com • Vol. 13 N. 25. 
dos ângulos de ligação em torno do nitrogênio são todos aproximadamente $360^{\circ}$, indicando uma geometria trigonal-planar e, portanto, uma hibridização $s p^{2}$. Desta forma, a maior estabilidade destas conformações pode ser explicada através de ressonância. No caso dos estados de transição, o somatório fica mais distante de $360^{\circ}$, e o nitrogênio apresenta geometria piramidal e uma hibridização intermediária entre $s p^{2}$ e $s p^{3}$. Comprova-se, assim, os confôrmeros que apresentam as ressonâncias mínimas, justificando serem os mais instáveis.

$\mathrm{Na}$ Figura 4, pode-se acompanhar a variação dos comprimentos das ligações $\mathrm{O}_{1}-\mathrm{C}_{2}$, $\mathrm{C}_{2}-\mathrm{N}_{3}$ e $\quad \mathrm{N}_{3}-\mathrm{C}_{4}$ obtidos para o $N$-metil- $N-(p-$ tolil)carbamato de terc-butila (8), através de cálculo semi-empírico AM1.

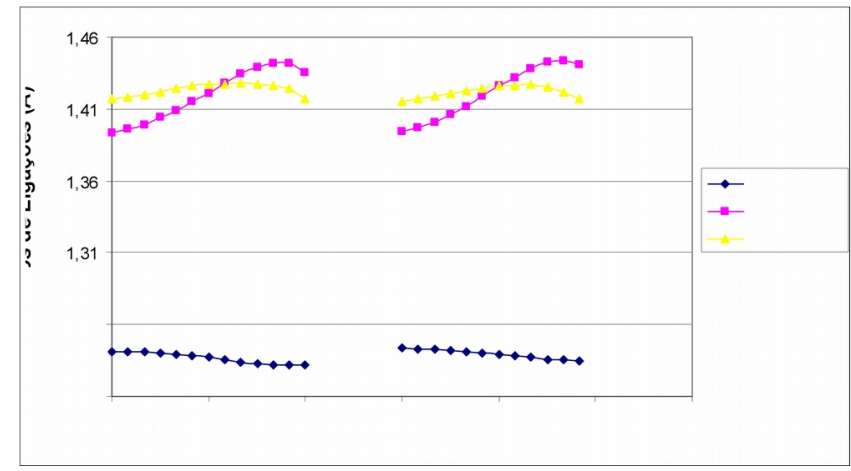

Figura 4. Comprimentos de ligações $(\AA) x$ diedro (graus): valores obtidos para o $N$-metil- $N-(p-$ tolil)carbamato de terc-butila (8), através de cálculo semi-empírico AM1 (PcSpartan Pro, versão 1.0.5).

O gráfico apresenta uma variação de aproximadamente $0,05 \AA$ na ligação $\mathrm{C}_{2}-\mathrm{N}_{3}$. Este efeito é atribuído ao fato de que, ao girar a ligação entre o nitrogênio e o carbono da carbonila, o par de elétrons do primeiro deixa de ser paralelo ao orbital $p$ de $\mathrm{C}_{2}$, diminuindo desta forma a ressonância. Uma variação equivalente não é observada nas demais ligações. O comportamento dos comprimentos de ligações, assim como o valor de energia da barreira rotacional (Figura 1) e a geometria do nitrogênio (Figura 3), se reproduzem para todos os demais compostos estudados.

Os resultados computacionais realizados pelo método semi-empírico AM1 indicaram maior estabilidade para as geometrias $\mathrm{E}$, com uma diferença mais pronunciada para carbamatos secundários $(1-5,11-15)$. Os resultados pelo método ab initio HF/6-31G* indicaram uma maior estabilidade para as geometrias $E$ em carbamatos secundários (1-5, 11-15) e Z em carbamatos terciários $(6-10,16-20)$. A Tabela 1 , a seguir, apresenta valores típicos das diferenças de energia obtidos para os $N$-fenilcarbamatos de terc-butila e metila.

Tabela 1. Diferenças de energias das geometrias $E$ e Z, obtidos através de cálculos semiempíricos e ab initio, para carbamatos secundários e terciários.

\begin{tabular}{|c|c|c|}
\hline Compostos & $\begin{array}{c}\Delta(\Delta \mathrm{H})_{E \rightarrow Z} \\
\left({\left.\mathrm{kcal} . \mathrm{mol}^{-1}\right)}^{\text {AM1 }}\right.\end{array}$ & $\begin{array}{c}\Delta \mathrm{E}_{T E \rightarrow Z} \\
\left(\mathrm{kcal} . \mathrm{mol}^{-1}\right) \\
\mathrm{HF} / 6-31 \mathrm{G}^{*}\end{array}$ \\
\hline 1 & 1,32 & 1,34 \\
\hline 2 & 1,43 & 2,13 \\
\hline 3 & 1,44 & 2,35 \\
\hline 4 & 1,51 & 2,53 \\
\hline 5 & 1,56 & 3,06 \\
\hline 6 & 0,46 & $-1,35$ \\
\hline 7 & 0,50 & $-1,18$ \\
\hline 8 & 0,50 & $-1,15$ \\
\hline 9 & 0,52 & $-0,94$ \\
\hline 10 & 0,56 & $-0,17$ \\
\hline 11 & 1,45 & 1,77 \\
\hline 12 & 1,44 & 2,21 \\
\hline 13 & 1,46 & 2,38 \\
\hline 14 & 1,50 & 2,59 \\
\hline 15 & 1,64 & 3,08 \\
\hline 16 & 0,59 & $-1,35$ \\
\hline 17 & 0,54 & $-1,19$ \\
\hline 18 & 0,78 & $-1,05$ \\
\hline 19 & 0,55 & $-0,83$ \\
\hline 20 & 0,65 & $-0,09$ \\
\hline
\end{tabular}

Em carbamatos secundários os dois grupos mais volumosos assumem orientação trans, tornando menor o impedimento espacial, podendo assim o anel manter a planaridade com a ligação entre o nitrogênio e a carbonila, aumentando a estabilidade da conformação $E$ por ressonância. Conforme citado anteriormente, o mesmo havia sido observado por Saito e colaboradores (1995) para $\mathrm{N}$-arilamidas. 


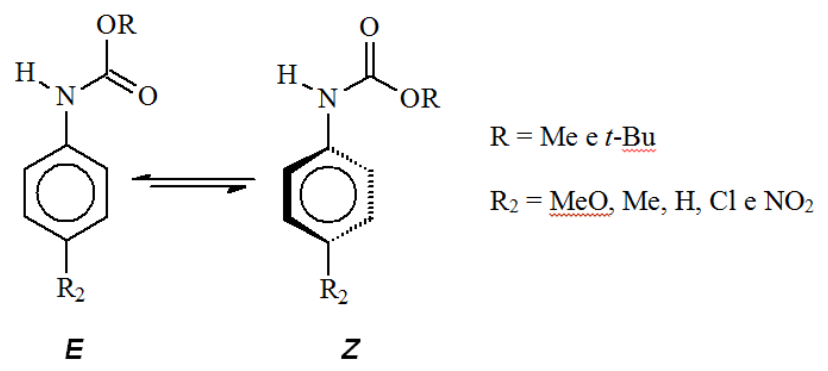

Esquema 5. Conformações de equilíbrio de carbamatos secundários.

No caso dos carbamatos terciários, as geometrias $\mathrm{E}$ e $\mathrm{Z}$ têm energias muito próximas, conforme o cálculo semi-empírico demonstrado na Tabela 1. A conformação $E$ possui interação espacial do $\mathrm{N}$-metil com a fenila, que a faz sair do plano da ligação entre o nitrogênio e a carbonila. Com isto não há mais a estabilização por ressonância como havia nos secundários. O cálculo ab initio, por sua vez, apresenta uma inversão do equilíbrio. Além da inclinação da fenila, que desestabiliza E, ainda ocorre a repulsão eletrônica entre o oxigênio da carbonila e a fenila. Cabe lembrar, que o oxigênio do grupo alcoxila é mais pobre em elétrons do que o da carbonila.

No processo rotacional da ligação entre o nitrogênio e a carbonila de amidas, o estado de transição preferencial é o $E T_{\text {anti. }} E T_{\text {syn }}$ é desestabilizado devido à repulsão dos pares isolados dos dois heteroátomos (Souza et al., 1996), conforme apresentado no Esquema 6.

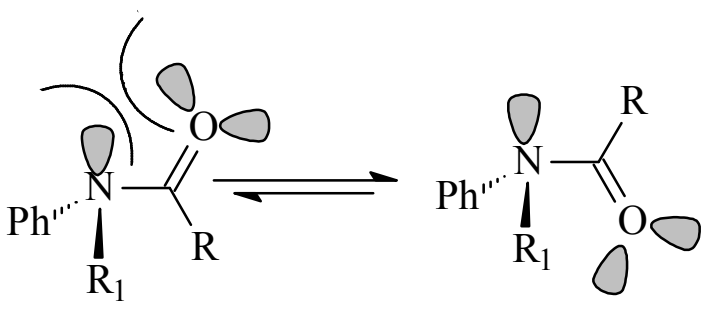
$\mathbf{E T}_{\text {syn }}$
$\mathbf{E T}_{a n t i}$

Esquema 6. Estados de transição de amidas.

Os resultados semi-empíricos AM1 indicaram $\mathrm{ET}_{\text {syn }}$ como estado de transição mais estável para os todos os carbamatos estudados. Já os cálculos ab initio HF/6-31G* indicaram $\mathrm{ET}_{\text {anti }}$ mais estável para os carbamatos secundários e para os carbamatos terciários não se obteve uma ordem. Estes resultados se devem ao fato de $\mathrm{ET}_{\text {syn }} \mathrm{e} \mathrm{ET}_{\text {anti }}$ possuírem energias muito próximas. Em carbamatos, qualquer que seja o estado de transição, sempre vai haver repulsão eletrônica, conforme apresentado no Esquema 7.

A conversão do rotâmero $E$ em $Z$, pode ocorrer por dois caminhos, seja através de $\mathrm{ET}_{\text {syn }}$, seja através de $\mathrm{ET}_{\text {anti. }} \mathrm{O}$ mesmo ocorre no caminho inverso, de tal forma que quatro diferentes barreiras podem ser definidas. A Tabela 2, a seguir, apresenta valores típicos.

Analisando a Tabela 3, verifica-se que no geral, as diferenças envolvendo $E \rightarrow E T_{\text {syn }}$ e $\mathrm{E} \rightarrow \mathrm{ET}_{\text {anti }}$ são as mais significativas, que as diferenças $Z \rightarrow E T_{\text {syn }}$ e $Z \rightarrow E T_{\text {anti. }}$.
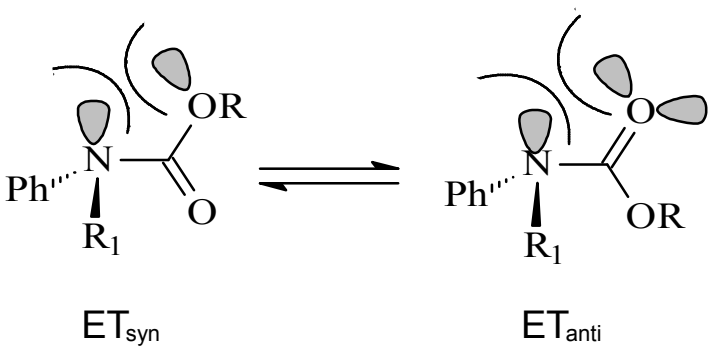

Esquema 7. Repulsão eletrônica dos pares de elétrons isolados dos heteroátomos nos estados de transição.

Tabela 2. Barreiras rotacionais de $\mathrm{N}$ arilcarbamatos obtidas por cálculo ab initio no conjunto de bases HF/6-31G*.

\begin{tabular}{|c|c|c|c|c|}
\hline $\begin{array}{c}\text { Compos- } \\
\text { tos }\end{array}$ & $\begin{array}{c}\Delta \mathrm{E}_{\mathrm{T}} \\
\left(E \rightarrow \mathrm{ET}_{s y n}\right) \\
\left(\mathrm{kcal} . \mathrm{mol}^{-1}\right)\end{array}$ & $\begin{array}{c}\Delta \mathrm{E}_{\mathrm{T}} \\
\left(E \rightarrow \mathrm{ET}_{\text {antit }}\right) \\
\left(\mathrm{kcal} . \mathrm{mol}^{-1}\right)\end{array}$ & $\begin{array}{c}\Delta \mathrm{E}_{\mathrm{T}} \\
\left(\mathrm{Z} \rightarrow \mathrm{ET}_{\text {syn }}\right) \\
\left(\mathrm{kcal} . \mathrm{mol}^{-1}\right)\end{array}$ & $\begin{array}{c}\Delta \mathrm{E}_{\mathrm{T}} \\
\left(\mathrm{Z} \rightarrow \mathrm{ET}_{\text {antit }}\right) \\
\left(\mathrm{kcal} . \mathrm{mol}^{-1}\right)\end{array}$ \\
\hline 1 & 17,06 & 15,92 & 15,72 & 14,58 \\
\hline 2 & 17,32 & 16,69 & 15,19 & 14,56 \\
\hline 3 & 17,30 & 16,82 & 14,95 & 14,47 \\
\hline 4 & 17,49 & 16,99 & 14,96 & 14,46 \\
\hline 5 & 16,88 & 15,76 & 13,82 & 12,71 \\
\hline 6 & 14,32 & 13,42 & 15,66 & 14,76 \\
\hline 7 & 13,81 & 14,29 & 14,98 & 15,47 \\
\hline 8 & 13,57 & 14,16 & 14,72 & 15,31 \\
\hline 9 & 13,57 & 14,16 & 14,51 & 15,10 \\
\hline 10 & 12,03 & 12,65 & 12,20 & 12,81 \\
\hline 11 & 17,28 & 16,02 & 15,52 & 14,25 \\
\hline 12 & 17,36 & 16,86 & 15,16 & 14,66 \\
\hline 13 & 17,30 & 16,60 & 14,92 & 14,22 \\
\hline 14 & 17,54 & 16,86 & 14,95 & 14,27 \\
\hline 15 & 16,95 & 15,62 & 13,87 & 12,54 \\
\hline 16 & 14,45 & 13,26 & 15,80 & 14,61 \\
\hline 17 & 13,93 & 14,16 & 15,12 & 15,35 \\
\hline
\end{tabular}




\begin{tabular}{rrrrr}
\hline $\mathbf{1 8}$ & 13,79 & 14,10 & 14,85 & 15,15 \\
\hline $\mathbf{1 9}$ & 13,86 & 14,16 & 14,69 & 14,99 \\
\hline $\mathbf{2 0}$ & 12,33 & 11,68 & 12,42 & 11,77 \\
\hline
\end{tabular}

O grupo alcoxila $\left(\mathrm{R}_{3}\right.$, conforme Esquema $3)$, praticamente não influencia as barreiras. Diferenças na barreira de secundários para terciários, entretanto, surgem devido a maior estabilidade do rotâmero $\mathrm{E}$ dos primeiros, por motivos os quais já foram discutidos.

Os carbamatos são estabilizados por ressonância. Esta estabilização é dependente do efeito eletrônico do grupo $R_{2}$ (Esquema 3). O Esquema 8 apresenta as estruturas de ressonância do rotâmero $\mathrm{E}$ de carbamatos secundários.<smiles>[R2]C(=O)N([R])c1ccc(CC)cc1</smiles>

a

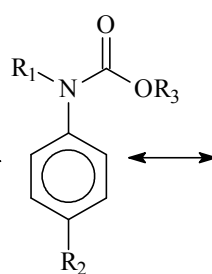

b

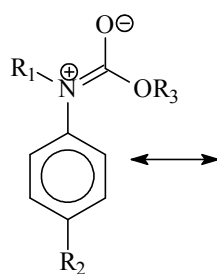

C

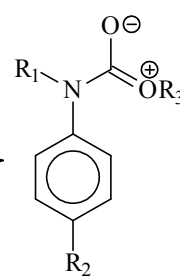

d
Esquema 8. Estruturas de ressonância de carbamatos.

Grupos doadores de elétrons ligados ao anel aumentam a participação da forma canônica c e diminuem a participação de a. No caso dos retiradores, a forma canônica a torna-se mais importante, com conseqüente diminuição na participação de c. Neste caso, um efeito compensa o outro, e não há uma maior influência do efeito eletrônico do grupo $R_{2}$ (Esquema3) na estabilidade do confôrmero. No rotâmero $E$ dos secundários e nas duas geometrias de equilíbrio dos terciários, por outro lado, o grupo arila encontra-se fora do plano da ligação entre o nitrogênio e a carbonila e a forma canônica $\mathbf{c}$ não existe. Uma menor participação da forma canônica a causada por um grupo retirador, leva a uma diminuição da ressonância e, portanto, da estabilidade do confôrmero. Então, pode-se concluir, que a estabilização das geometrias de equilíbrio segue a seguinte ordem: $\mathrm{NO} 2<\mathrm{Cl}<\mathrm{H}$ $<\mathrm{Me}<\mathrm{MeO}$, com exceção de $\mathrm{E}$ em carbamatos secundários, os quais não são influenciados pela natureza do grupo $\mathrm{R}_{2}$.

Por método experimental (Deetz et al., 2002) a barreira rotacional de $N$ - alquilcarbamatos é normalmente em torno de 16 kcal.mol ${ }^{-1}$, sendo cerca de 1 a $4 \mathrm{kcal}^{\mathrm{mol}}{ }^{-1}$ mais baixo que as amidas estruturalmente relacionadas. No caso dos $\mathrm{N}$-fenilcarbamatos a barreira rotacional é mais baixa, em torno de 12,5 kcal.mol ${ }^{-1}$. Cálculos realizados pela metodologia HF/6-31G* apresentam os valores de barreira em torno de 13,7 kcal.mol ${ }^{-1}$. Os resultados obtidos por método semi-empírico demonstram uma relação de aproximadamente $40 \%$ dos valores obtidos pelo método $a b$ initio. A comparação dos resultados computacionais com os experimentais (Smith et al. 2004) está apresentada na Tabela 3.

Tabela 3. Valores das barreiras rotacionais $\left(\mathrm{kcal}^{\mathrm{mol}} \mathrm{H}^{-1}\right)$ de carbamatos de terc-butila parasubstituídos.

\begin{tabular}{cccc}
\hline $\begin{array}{c}\text { Compos- } \\
\text { tos }\end{array}$ & $\begin{array}{c}\text { Experimen- } \\
\text { tal }\end{array}$ & $\begin{array}{c}\mathrm{AM} 1 \\
\left(E \rightarrow \mathrm{ET}_{\text {syn }}\right)\end{array}$ & $\begin{array}{c}\mathrm{HF} / 6-31 \mathrm{G}^{*} \\
\left(E \rightarrow \mathrm{ET}_{\text {syn }}\right)\end{array}$ \\
\hline $\mathbf{6}$ & 13,2 & 5,3 & 14,3 \\
\hline $\mathbf{7}$ & 12,5 & 5,1 & 13,8 \\
\hline $\mathbf{8}$ & 12,3 & 5,0 & 13,6 \\
\hline $\mathbf{9}$ & 12,3 & 5,0 & 13,6 \\
\hline
\end{tabular}

\section{CONCLUSÕES}

1. Estudos do equilíbrio conformacional realizados pelo método semi-empírico AM1, demonstraram maior estabilidade para a geometria de equilíbrio $E$ com uma diferença mais pronunciada para carbamatos secundários. Cálculos ab initios HF/6-31G* confirmaram esses resultados para carbamatos secundários, mas demonstram o inverso para carbamatos terciários.

2. Estudos da estabilidade do Estado de Transição pelo método semi-empírico, indicaram maior estabilidade para $\mathrm{ET}_{\text {syn. }}$ Estudos ab initio mostraram o $\mathrm{ET}_{\text {anti }}$ como estado de transição mais estável para carbamatos secundários, mas não indicaram qualquer tendência no caso dos terciários.

3. As geometrias de equilíbrio apresentam o nitrogênio planar indicando uma hibridização $s p^{2}$. Os estados de transição apresentam o nitrogênio piramidal, com hibridização intermediária entre $s p^{2}$ e $s p^{3}$.

4. Grupos doadores de elétrons ligados ao anel aromático estabilizam as geometrias de equilíbrio 
de $\quad \mathrm{N}$-arilcarbamatos terciários. Grupos retiradores de elétron exercem o efeito contrário. $\mathrm{O}$ mesmo efeito é observado no rotâmero $Z$ dos análogos secundários, mas a estabilidade da geometria $E$ não é influenciada pelo efeito eletrônico do substituinte ligado à arila.

5. Os grupos alcoxila não influenciam na energia da barreira.

6. Os resultados semi-empíricos demonstraram uma relação de aproximadamente $40 \%$ do valor da barreira rotacional em relação aos ab initio.

7. Os cálculos ab initio estimaram as barreiras rotacionais no intervalo de 13 a $17 \mathrm{kcal}^{\mathrm{mol}}{ }^{-1}$, muito próximos a valores experimentais descritos na literatura (Deetz et al., 2002).

\section{REFERÊNCIAS:}

1. Avalos, M., Babiano, R., Barneto, J. L., Bravo, J. L., Cintas, P., Jiménez, J. L. e Palacios, J. C. J. Org. Chem. 2001, 66, 7275.

1. Deetz, M. J., Forbes, C. C., Jonas, M., Malerich, J. P., Smith, B. D., Wiest, O. J. Org. Chem. 2002, 67, 3949.

2. Dewar, M. J. S., Zoebisch, E. G., Healy, E. F., Stewart, J. J. P. J. Am. Chem. Soc. 1985, 107, 3902.

3. Eliel, E. L. e Wilen, S. H., Sterochemistry of Compounds Organics, Wiley, Nova lorque,1994.

4. Fischer, G. Chem. Soc. Rev. 2000, 119.

5. Fontoura, L. A. M., Rigotti, I. J. C. e Correia, C. R. D. J. Molec. Struct. 2002, 609, 73.

6. Gálvez, J., Sánchez, J. I. L., Guirado, A. Comput. Theor. Chem., 2015, no prelo (10.1016/j.comptc.2015.06.020).

7. Ilieva, S., Hadjieva, B e Galabov, B. J. Org. Chem. 2002, 67, 6210.
8. Kim, N. C., Kim, J. H., Kim, J. H., Nam, S. W., Jeon, B. S., \& Kim, Y. J. J. Ind. Eng. Chem., 2015, $28,236$.

9. Latelli, N., Ouddai, N., Arotçaréna, M., Chaumont, P., Mignon, P., Chermette, H. Comput. Theor. Chem., 2014, 1027, 39.

10. Lauvergnat, D. e Hiberty, P. C. J. Am. Chem. Soc. 1997, 119, 9478.

11. Liang, P., Bao, H., Yang, J., Zhang, L., \& Chen, G. Carbon, 2016, 97, 25.

12. Mizushima, S., Simanouti, T., Nagakura, S., Kuratani, K., Tsuboi, M., Baba, H., Fujioka, O. J. Am. Chem. Soc., 1950, 72, 3490.

13. Montazer, M., Nia, Z. K. Mater. Sci. Eng., C, 2015, 56, 341.

14. Moreno, H. M., Jacq, C., Montero, M. P., Gómez-Guillén, M. C., Borderías, A. J., Mørkøre, T. Food Chem., 2016, 190, 856.

15. Ortega, P. R., Montejo, M., Márquez, F., González, J. L. J. Mol. Graph. Model. 2015, no prelo (10.1016/j.jmgm.2015.05.011)

16. Pawar, D. M., Khalil, A. A., Hooks, D. R., Collins, K., Elliott, T., Stafford, J., Noe, E. A. J. Am. Chem. Soc. 1998, 120, 2108.

17. Saito, S., Toriumi, Y., Tomioka, N. e Itai, A. J. Org. Chem. 1995, 60, 4715.

18. Smith, B. D., Goodenough-Lashua, D. M., D'Souza, C. J. E., Norton, K. J., Schmidt, L. $M$ e Tung, J. C. Tetrahedron Lett., 2004, 45, 2747.

19. Souza, W. F., Kambe, N. e Sonoda, N. J. Phys. Org. Chem. 1996, 9, 179.

20. Sun, L., Jia, L., Xie, X., Xie, K., Wang, J., Liu, J., Zhang, G., Dai, G., Wang, J. Food Chem., 2015, 192, 313.

21. Taha, A.N. Crawford, S.M.N. True, N.S. J. Am. Chem. Soc. 1998, 120, 1934.

22. Wiberg, K. B. e Breneman, C. M. J. Am. Chem. Soc. 1992, 114, 831. 